\title{
Potential hazards of intraoperative cholangiography in patients with infected bile
}

\author{
N J LYGIDAKIS* \\ From the Saint Panteleman Hospital, Athens, Greece
}

SUMMARY To assess the relation of bacteraemia to intraoperative cholangiography 194 patients with choledocholithiasis who underwent choledochotomy and who had intraoperative cholangiography through the cystic duct are presented in this study. It has been shown that, in the presence of infected bile, intraoperative cholangiography without manometric control is hazardous, provoking a high incidence of bacteraemia and associated with considerable mortality and morbidity rate. It is suggested that intraoperative cholangiography should be carried out under manometric control, and that it should be used only with extreme care in patients who are likely to have biliary infections.

The incidence and significance of infected bile has been previously studied in patients with benign or malignant obstruction of the extrahepatic biliary tree. ${ }^{1-3}$

It has been shown that operation in the presence of bacteria in the bile is associated with a higher morbidity and mortality, and the incidence of cholangitis after percutaneous transhepatic cholangiography, endoscopic retrograde cholangiopancreatography, and postoperative cholangiography have been well documented. ${ }^{4-8}$

Little has been reported, however, concerning the potential hazards associated with intraoperative cholangiography. ${ }^{9}$ This study examines two different methods of intraoperative cholangiography in relation to the presence of infected bile.

\section{Methods}

PATIENTS

One hundred and ninety-four consecutive patients with cholelithiasis and choledocholithiasis are presented who underwent cholecystectomy and common bile duct exploration and T-tube drainage during the period 1975-80 in Athens, Greece. There were 77 males and 117 females (age range 8 to 79 years, mean 58 years). The length of history before operation was from one to 19 years (mean nine years).

* Present address: Royal Postgraduate Medical School, Hammersmith Hospital, London W12 0HS.

Received for publication 25 January 1982
The pain was the predominant symptom, localised in the right upper quadrant and radiating to the back (184 patients). Attacks of fever consistent with diagnosis of recurrent cholangitis were reported by 42 patients. On admission 49 patients presented with jaundice (Table 1). No patient was admitted with clinical evidence of bacteraemia.

In all 194 patients intraoperative cholangiography using $45 \%$ Hypaque as the contrast medium was carried out via a cannula inserted into the cystic duct.

The patients were divided into two groups: Group A (90 patients)

In this group cholangiography was carried out by manual injection of contrast medium using a $20 \mathrm{ml}$

Table 1

\begin{tabular}{lll}
\hline & $\begin{array}{l}\text { Group A } \\
(90)\end{array}$ & $\begin{array}{l}\text { Group B } \\
(104)\end{array}$ \\
\hline Male & 32 & 45 \\
Female & 58 & 59 \\
Age (yr) & $28-74$ & $28-79$ \\
& $($ mean 57) & (mean 58) \\
Length of history (yr) & $1-15$ & $2-19$ \\
Previous attacks of cholangitis & 15 & (mean 9) \\
Jaundice at admission & 17 & 27 \\
Pain & 88 & 32 \\
Anorexia/weight loss & 33 & 96 \\
Blood culture + (intraoperative) & 1 & 44 \\
Bile culture (intraoperative) & & 2 \\
\hline
\end{tabular}

* Twelve patients of group A and 25 patients of group B with positive bile cultures were jaundiced at admission. 
syringe, three films being exposed after injection of 3,5 , and $10 \mathrm{ml}$ contrast material.

Group B (104 patients)

In this group cholangiography was performed with a simple and easy-to-use apparatus (debitomanometer), the common bile duct being filled with contrast medium at a pressure of $15 \mathrm{~cm}$ water. A mean of $20 \mathrm{ml}$ contrast medium was used. Three cholangiograms were taken at this level of pressure.

No method of selection was used in the choice of the procedure. The first 90 patients were subjected to manual injection of contrast medium at uncontrolled pressure and the subsequent 104 patients to manometrically controlled injection. Subsequent comparison, however, of groups $\mathrm{A}$ and $B$ proved them to be comparable in terms of age, sex, and length of history; there was also no significant difference between the groups in the number of patients presenting with jaundice, a history of previous attacks of cholangitis, or the incidence of pain, anorexia, or weight loss (Table 2). In addition, the preoperative incidence of abnormal liver function tests, raised amylase or raised creatinine levels were also found to be comparable, as were the incidence of intraoperative positive bile and blood cultures.

All patients were given a preoperative antibiotic, starting 12 hours before operation and continuing for five postoperative days (gentamicin $80 \mathrm{mg}$ eight hourly and ampicillin $1 \mathrm{~g}$ qds).

\section{INTRAOPERATIVE STUDIES}

After opening the abdomen bile samples were withdrawn by needle puncture from the gall bladder and common bile duct for aerobic and anaerobic culture and simultaneously $10 \mathrm{ml}$ venous blood was drawn into a sterile syringe for blood culture.

During operation the diameter of the common bile duct was measured above cystic duct. Common bile duct stones were then assessed, those that were yellow or brown, large, soft, and crushable stones being characterised as primary stones. ${ }^{4}$

Table 2 Relation of bile infection to age in 194 patients with choledocholithiasis

\begin{tabular}{lcc}
\hline & \multicolumn{2}{c}{ Patients } \\
\cline { 2 - 3 } Age & No. & No. with infected bile \\
\hline $20-30$ & 17 & 1 \\
$31-40$ & 35 & 2 \\
$41-50$ & 32 & 9 \\
$51-60$ & 81 & 60 \\
$61-70$ & 21 & 20 \\
$71-80$ & 8 & 7 \\
Total & 194 & 99 \\
\hline
\end{tabular}

POST-OPERATIVE STUDIES

Blood cultures were carried out in all the patients six hours after surgery and, in those patients with bacteraemia, were repeated daily for the next five postoperative days. Antibiotic treatment was adjusted according to the sensitivity tests obtained.

\section{Results}

Liver function tests were found to be abnormal in 49 patients. The amylase was not increased, although it was found to be mildly raised in nine cases. Creatinine was raised in seven patients all of whom had presented with jaundice. In patients with clinically evident jaundice there was always an increase in the level of bilirubin and of alkaline phosphate.

The operative findings revealed bile infection to be much more common in patients with dilated common bile ducts, in patients with primary common bile duct stones, and in those with a long history of symptoms and of advanced age.

Bile culture was positive in 99 patients (Table 1). An average of three organisms was cultured per patient, with as many as seven organisms being cultured in the bile of one individual. Eighty per cent of the organisms cultured were aerobes with gram negative organisms being twice as common as the gram positive organisms. Escherichia coli and Klebsiella pneumoniae were the most common Gram negative organisms and enterococcus the Gram positive organism cultured most frequently, being found in the bile of 39 patients. Twenty per cent of the bacteria cultured were anaerobic, however, though bacteroides were the most commonly isolated species.

There was increased evidence of bile infection in patients with a long history of symptoms, advanced age, dilated common bile duct, or the finding of primary common bile duct stones. There was no difference between the two groups in respect of these findings (Tables 2, 3, and 4).

Table 3 Relation of bile infection to length of symptoms in 194 patients with choledocholithiasis

\begin{tabular}{lcc}
\hline \multirow{2}{*}{$\begin{array}{l}\text { Lenght of symptoms } \\
\text { (yr) }\end{array}$} & \multicolumn{2}{l}{ Patients } \\
\cline { 2 - 3 } & No. & No. with infected bile \\
\hline $1-5$ & 37 & 2 \\
$5-10$ & 102 & 56 \\
$10-15$ & 35 & 25 \\
$15-20$ & 20 & 16 \\
Total & 194 & 99 \\
\hline
\end{tabular}


Table 4 Association of bile infection with common bile duct diameter, histology and primary stones

\begin{tabular}{lrrll}
\hline & \multicolumn{3}{l}{ Patients (no.) } \\
\cline { 2 - 5 } CBD diam & & $\begin{array}{l}\text { With } \\
\text { primary } \\
\text { stones }\end{array}$ & $\begin{array}{l}\text { With } \\
\text { histological } \\
\text { changes }\end{array}$ & $\begin{array}{l}\text { With } \\
\text { bile } \\
\text { infection }\end{array}$ \\
\hline$<1 \cdot 5$ & 80 & 7 & 0 & 12 \\
$>1 \cdot 5$ & 80 & 19 & 10 & 49 \\
$>2$ & 20 & 16 & 16 & 19 \\
$>3$ & 10 & 9 & 8 & 10 \\
$>4$ & 9 & 8 & 7 & 9 \\
Total & 199 & 59 & 41 & 99 \\
\hline
\end{tabular}

Intraoperative blood culture was positive in three patients; one in group A and two in group B. Six hours after operation, blood cultures revealed bacteraemia in 29 patients including all three with positive blood cultures before operation. All patients with bacteraemia had a positive bile culture at the time of operation. The incidence of bacteraemia was higher those presenting with jaundice at operation.

There was a marked difference between the incidence of postoperative bacteraemia between groups A and B. Bacteraemia was found in $23.3 \%$ of patients in group $A$ and $7.6 \%$ of those in group B. In patients with infected bile at the time of operation $51 \%$ were found to have postoperative bacteraemia within group A and $7 \%$ of patients with group B $(\mathrm{p}<0.001)$. In addition, bacteraemia was more common in jaundiced patients of group A $(66.9 \%)$ compared with those presenting with jaundice in group B $(25 \%)(\mathrm{p}<0.001)$.

Two postoperative deaths occurred, both in group

Table 5 Incidence of postoperative mortality, morbidity, and bacteraemia in 194 patients who had intraoperative cholangiography and of bacteraemia in 32 patients who presented with jaundice

\begin{tabular}{llllll}
\hline & $\begin{array}{l}\text { Wound } \\
\text { infection } \\
\text { (no.) }\end{array}$ & $\begin{array}{l}\text { Respir- } \\
\text { atory } \\
\text { infection } \\
\text { (no.) }\end{array}$ & $\begin{array}{l}\text { Deaths } \\
\text { (no.) }\end{array}$ & $\begin{array}{l}\text { Bacter- } \\
\text { aemia } \\
\text { (no.) }\end{array}$ & $\begin{array}{l}\text { Bemia } \\
\text { with } \\
\text { jaundice } \\
\text { (no.) }\end{array}$ \\
\hline $\begin{array}{l}\text { Group A } \\
90 \text { patients }\end{array}$ & 16 & 14 & 2 & 21 & $7 / 17$ \\
$\begin{array}{l}\text { Group B } \\
104 \text { patients }\end{array}$ & 3 & 2 & 0 & 8 & $5 / 32$ \\
\hline
\end{tabular}

Twelve patient in group $\mathbf{A}$ and 25 patients in group B had positive bile cultures, and were admitted with jaundice
A patients with bile infection and bacteraemia (Table 5).

Postoperative morbidity was also higher in the presence of bile infection and bacteraemia and occurred more often in patients of group A $(p<0.001)$. Postoperative bacteraemia was associated with fever and rigors in 14 patients. In nine there was associated hypotension and oliguria. It is important, however, to note that positive blood cultures were obtained in six patients who showed no evidence of clinical symptoms.

The organisms cultured from the blood were the same as those obtained from the previously cultured bile. Bacteraemia persisted for two days in 14 patients, for three days in nine patients, and for four days in four patients. The remaining two patients deteriorated clinically and developed bacteraemic shock and died on the sixth and seventh postoperative days respectively. In both instances the organism isolated was the anaerobe Bacteroides fragilis. A variety of other organisms was isolated from the blood in the remaining 27 patients. Sensitivity tests from the blood and the bile revealed gentamicin to be effective in $94 \%$ of the Gram negative aerobes, cephalothin in $60 \%$, and ampicillin in $31 \%$. Eighty per cent of the organisms were sensitive to a combination of ampicillin and gentamicin.

\section{Discussion}

The results indicate that the incidence of bile infection in patients with choledocholithiasis is related to age, the length of history, presence of jaundice, presence of primary common bile duct stones, and a dilated biliary tree. These features are in accordance with the findings of others. ${ }^{1-3}$

Experimental evidence suggests that a provocative factor in the development of bacteraemia in patients with infected bile may be an increase of intraductal pressure and that, in the presence of infected bile, any increase of pressure over $15 \mathrm{~cm}$ of water can easily initiate reflux of bacteria from the biliary system to the blood stream. It has also been demonstrated that a direct communication exists between the cannaliculae of the terminal bile duct and the liver sinusoid ${ }^{1011}$ and these findings are supported by demonstration of reflux of radioactively labelled albumin and gold injected into the obstructed biliary duct of experimental animals. ${ }^{12}$ In addition, it has been shown that radioactively labelled bacteria reflux into the blood stream if the pressure is only slightly higher than the secretory pressure of the liver $(15 \mathrm{~cm}$ of water) and that when biliary ductal pressures were raised above this level bacteria appeared rapidly in both the blood and the 
lymph, concentrations of bacteria in both systems being directly proportional to the bile duct pressure. ${ }^{13}$

Indeed, in this study there is a significant difference in the incidence of bacteraemia and morbidity between patients of group $\mathrm{A}$ in whom the injection pressure was high and in those of group B in whom it was controlled. Both deaths occurred in patients in group $\mathrm{A}$. These findings suggest a potential hazard of intraoperative cholangiography if carried out in such a way as to raise the intraductal biliary pressure in an uncontrolled fashion.

As all patients had intraoperative antibiotic cover it would seem that such prophylactic measures are only partly effective in inhibiting bacteraemia.

It would also seem that operative cholangiography should be carried out by a technique which allows control of the intraductal pressure and, even then, it should be cautiously used in patients in whom it might be reasonable to assume biliary infection.

\section{References}

1 Goswitz ST. Bacteria and biliary tract disease. Am J Surg 1974; 128: 644-6.

2 Nielson ML, Justesen T. Anerobic bacteriological studies in biliary tract disease. Scand J Gastroenterol 1976; 11: 437-46.

3 Keighley MRB. Micro-organisms in the bile. A preventable cause of sepsis after biliary surgery. Ann $R$ Coll Surg Engl 1977; 59: 328-34.

4 Lygidakis NJ. Incidence of bile infection in biliary calculous disease. Am J Gastroenterol. 1982; 77: 12-16.

5 Roudall PE, Hutchinson GH. Wound infection after biliary tract surgery. Br J Clin Pract 1980; 34: 200-02.

6 Lefrock J, Ellis CA, Tarchik SB et al. Transient bacteraemia associated with percutaneous liver biopsy $J$ Infect Dis 1975; 732: suppl 5: 104.

7 Okuda K, Tanikaua K, Emera T et al. Nonsurgical percutaneous transhepatic cholangiography: diagnostic significance in problems of the liver. Am J Dig Dis 1974; 19: 21-7.

8 Bilbao HK, Dotter CT, Lee TG. Complications of ERCP: A study of 10,000 cases. Gastroenterology 1976; 70: 314-9.

9 Martensson K. Grave ascending cholangitis following cholangiography carried out through the cystic duct infected with E. coli. Acta Chir Scand 1946; 94: 1-8.

10 Mixer HW, Rigler LG, Gonzales-Odove MV. Experimental studies on biliary regurgitation during cholangiography Gastroenterology 1947; 9: 64-71.

11 Edlund Y, Hazon V. Demonstration of the close relationship between bile capillaries and sinusoid walls. Acta Anat 1953; 17: 105-11.

12 Hultborn A, Jacobson B, Rosengreen B. Cholangiovenous reflex during cholangiography. Acta Chir Scand 1962; 123: 316-21.

13 Huaug T, Bass JA, Williams RD. The significance of biliary pressures in cholangitis Arch Surg 1972; 98: 629-32. 